\title{
Transition between chaotic and stochastic universality classes of kinetic roughening
}

\author{
Enrique Rodríguez-Fernández $\odot^{*}$ and Rodolfo Cuerno $\oplus^{\dagger}$ \\ Departamento de Matemáticas and Grupo Interdisciplinar de Sistemas Complejos (GISC), \\ Universidad Carlos III de Madrid, Avenida de la Universidad 30, 28911 Leganés, Spain
}

(Received 24 July 2020; accepted 16 February 2021; published 2 March 2021)

\begin{abstract}
The dynamics of nonequilibrium spatially extended systems are often dominated by fluctuations, e.g., due to deterministic chaos or intrinsic stochasticity. This reflects into generic scale invariant or kinetic roughening behavior that can be classified into universality classes defined by critical exponent values and by the probability distribution function (PDF) of field fluctuations. Suitable geometrical constraints are known to change secondary features of the PDF while keeping the values of the exponents unchanged, inducing universality subclasses. Working on the Kuramoto-Sivashinsky equation as a paradigm of spatiotemporal chaos, we show that the physical nature of the prevailing fluctuations (chaotic or stochastic) can also change the universality class while respecting the exponent values, as the PDF is substantially altered. This transition takes place at a nonzero value of the stochastic noise amplitude and may be suitable for experimental verification.
\end{abstract}

DOI: 10.1103/PhysRevResearch.3.L012020

Generic scale invariance (GSI) describes the behavior of many spatially extended nonequilibrium systems in which driving and dissipation act at comparable rates, such that strong correlations build up whose space-time behavior lacks characteristic scales [1]. Hence, they are analogous to equilibrium critical systems, a crucial difference being that parameter tuning is not required for criticality in GSI [2]. Thus, GSI is one of the forms in which critical dynamics [3] is being recently generalized to nonequilibrium contexts as assessed in physical and nonphysical systems, from quantum matter [4,5] to living [6] or social [7] systems.

A key player in recent advances on the understanding of driven systems displaying GSI [8-12] is the celebrated Kardar-Parisi-Zhang (KPZ) equation for the height $h(x, t)$ of an interface at a substrate position $x \in \mathbb{R}^{d}$ and time $t$ [13], subject to fluctuations, namely (we henceforth set $d=1$ ),

$$
\begin{gathered}
\partial_{t} h=v \partial_{x}^{2} h+\frac{\lambda}{2}\left(\partial_{x} h\right)^{2}+D \eta, \\
\left\langle\eta(x, t) \eta\left(x^{\prime}, t^{\prime}\right)\right\rangle=\delta\left(x-x^{\prime}\right) \delta\left(t-t^{\prime}\right),
\end{gathered}
$$

where $\nu, D>0$, and $\lambda$ are parameters, and $\eta(x, t)$ is zeroaverage Gaussian noise. Within the physical image of evolving interfaces, the GSI behavior displayed by the KPZ equation and related stochastic systems is termed kinetic roughening [8,9]. In analogy with equilibrium critical dynamics, it can be classified into universality classes, which

\footnotetext{
*enrodrig@math.uc3m.es

†cuerno@math.uc3m.es
}

Published by the American Physical Society under the terms of the Creative Commons Attribution 4.0 International license. Further distribution of this work must maintain attribution to the author(s) and the published article's title, journal citation, and DOI. are determined by the values of critical exponents and by the probability distribution function (PDF) of, say, height fluctuations [10-12]. Indeed, the one-dimensional (1D) KPZ universality class has been recently identified in the fluctuation dynamics of a wide range of low-dimensional, strongly correlated systems, from thin-film growth, random polymers, and randomly stirred fluids [8,9] to active matter [14], quantum entanglement [15], and spatiotemporal chaos [16], to cite a few.

Based on exact solutions of the one-dimensional (1D) KPZ equation, Eq. (1), and related discrete models (see Refs. [10-12] for reviews), a particularly rich structure is being elucidated for GSI universality classes. To begin with, critical exponent values are known not to unambiguously identify a given class. Indeed, explicit examples have been reported of linear height equations with time- [17] or space[18] correlated noise, which feature the 1D KPZ scaling exponents but which cannot be in this universality class, their field PDF being Gaussian, while for the nonlinear 1D KPZ equation it is a member of the Tracy-Widom (TW) PDF family [10-12].

Moreover, while keeping the same values of the scaling exponents, universality subclasses exist of the 1D KPZ class, which differ by the flavor of the precise TW PDF which occurs: For example, for globally flat (curved) interfaces growing from a straight line (point), it corresponds to the largest eigenvalue TW distribution of random matrices in the Gaussian orthogonal (unitary) ensemble [GOE (GUE)] [10-12]. Equivalently, finite systems whose size decreases (increases) linearly with time display TW-GOE (GUE) statistics [19-21], while analogous transitions have been assessed for changes in the background topology [22] or in the rate of system-size change [23]. Furthermore, the existence of universality subclasses induced by similar changes in geometrical constraints carries over to the main linear [24] and nonlinear [25] universality classes of kinetic roughening other 
than KPZ, making this a robust trait of (this type of) criticality far from equilibrium.

However, in all these cases the universality subclasses sharing scaling exponent values differ by some global geometrical or topological condition on the system size or background metric, the existence of alternative mechanisms which likewise control the field PDF remaining uncertain. In this Letter, we demonstrate the physical nature of the prevailing system fluctuations as one such mechanism. Specifically, by considering the 1D Kuramoto-Sivashinsky (KS) equation [26,27] for a scalar field $u(x, t)$, which reads

$$
\partial_{t} u=-v_{0} \partial_{x}^{2} u-\kappa_{0} \partial_{x}^{4} u+\lambda_{0} u \partial_{x} u+\left(D_{0}+\tilde{D}_{0} \partial_{x}\right) \eta,
$$

where $\nu_{0}, \kappa_{0}, D_{0}, \tilde{D}_{0}>0$, and $\lambda_{0}$ are parameters, and $\eta$ is as in Eq. (2), we show (for $D_{0}=0, \tilde{D}_{0} \neq 0$ ) that the kinetic roughening behavior which it displays is characterized by critical exponent values which are noise independent, while the field PDF is non-Gaussian (Gaussian) for low (large) noise values, corresponding to dynamics dominated by chaotic (stochastic) fluctuations. This transition in the universality class occurs at a nonzero noise amplitude $\tilde{D}_{0}$ and might be observable in suitable experimental contexts.

The deterministic $\left(D_{0}=\tilde{D}_{0}=0\right) \quad \mathrm{KS}$ equation is a paradigm of spatiotemporal chaos (SC) [28,29], where it has become a benchmark to assess novel concepts and tools, e.g., reservoir computing [30,31]. Either Eq. (3) proper or the equally ubiquitous version of the deterministic KS equation [26,27], satisfied by $h(x, t)=\int_{0}^{x} u(y, t) d y$ [see Eq. (5) below], both provide physical models in many different contexts, from liquid flow down inclines [32,33] to solidification [34]. The stochastic equation for $h$ has been derived in, e.g., epitaxial growth [35,36], ion-beam sputtering [37,38], or diffusionlimited growth [39]; in Ref. [40] we derive Eq. (3) for a falling liquid film under thermal fluctuations [41,42].

The large-scale behavior of the deterministic KS equation, Eq. (3) with $D_{0}=\tilde{D}_{0}=0$, is known to remarkably coincide $[43,44]$ with that of the stochastic Burgers equation, whose GSI exponents [45] and field PDF [18,46] are known. Likewise, the deterministic [16,47] and stochastic KS equations for $h[48,49]$ are both in the KPZ universality class. However, how and if the nature of the fluctuations, whether deterministic chaos or stochastic noise, reflects into the GSI behavior have remained overlooked thus far.

We begin by investigating in full detail the universality class of Eq. (3) for the deterministic case and the stochastic cases with conserved $\left(D_{0}=0, \tilde{D}_{0} \neq 0\right)$ and nonconserved $\left(D_{0} \neq 0, \tilde{D}_{0}=0\right)$ noise. Note that, as seminally argued for by Yakhot [43], Eq. (3) is expected to renormalize at large scales into an effective stochastic Burgers equation,

$$
\partial_{t} u=v \partial_{x}^{2} u+\lambda u \partial_{x} u+\left(D+\tilde{D} \partial_{x}\right) \eta,
$$

where notably $v>0$, rendering asymptotically irrelevant the biharmonic term in Eq. (3). Moreover, the noise in the effective equation, Eq. (4), respects the conservation law expressed by Eq. (3). That is, if the bare equation is deterministic or has conserved noise, then $D=0, \tilde{D} \neq 0$, while if the bare noise is nonconserved, so is the effective noise, and thus $D \neq 0$, $\tilde{D}=0$.

We have performed numerical simulations of Eq. (3) using the implementation of the pseudospectral method [50-54]

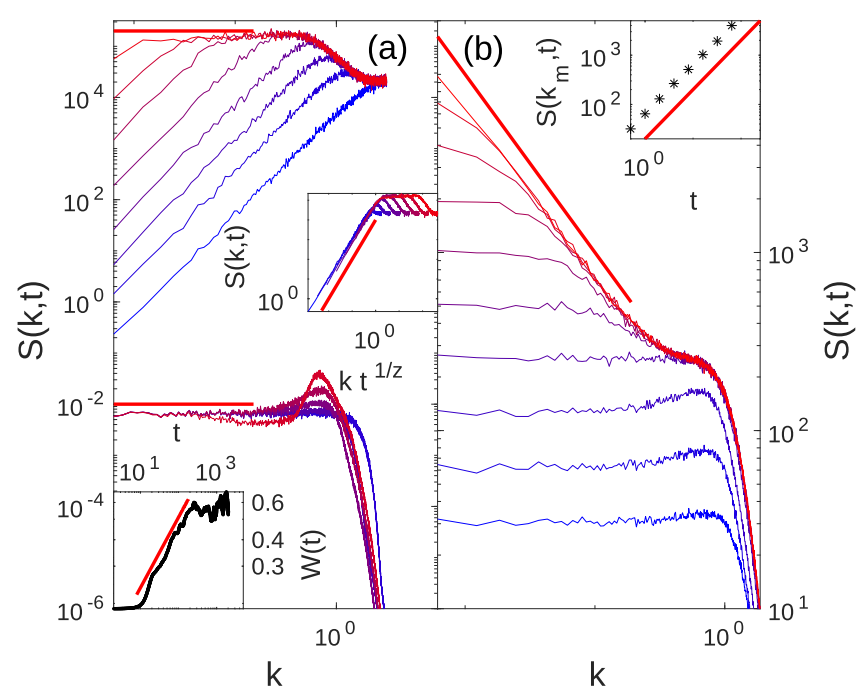

FIG. 1. Time evolution of $S(k, t)$ from numerical simulations of Eq. (3) for different noise conditions: (a) deterministic case $\left(D_{0}=\right.$ $\tilde{D}_{0}=0$; bottom) and conserved noise $\left(D_{0}=0, \tilde{D}_{0}=1\right.$; top). Top inset: data collapse for $\alpha_{\mathrm{cn}}=-1 / 2, z_{\mathrm{cn}}=3 / 2$. Bottom inset: time evolution of the roughness of $h(x, t)=\int_{0}^{x} u(y, t) d y$; the straight line has slope $\beta_{\mathrm{nc}}=\left(\alpha_{\mathrm{cn}}+1\right) / z_{\mathrm{cn}}=1 / 3$; (b) nonconserved noise $\left(D_{0}=1, \tilde{D}_{0}=0\right)$. Inset: time evolution of $S\left(k_{m}, t\right)$ for the smallest $k$ value, $k_{m}$; the straight line has slope $2 \alpha_{\mathrm{nc}}+1=z_{\mathrm{nc}}=1$. For all panels, averages are over 100 realizations, time increases from blue to red, and the slope of the red solid line for small $k$ is $-(2 \alpha+1)$ for $\alpha=\alpha_{\mathrm{cn}}=-1 / 2\left[\alpha_{\mathrm{nc}}=0\right]$ in panel (a) [(b)].

proposed in Ref. [55] and periodic boundary conditions for system size $L=2048$. Initial conditions are random (with $10^{-5}$ amplitude) for the deterministic case and zero otherwise. Parameters are fixed to $v_{0}=\kappa_{0}=1, \lambda_{0}=10$, and spacetime discretization steps $\delta x=1$, and $\delta t \in[0.01,0.05]$. Under GSI conditions [8,9], the scaling exponents characterizing the universality class can be readily identified in the evolution of the field roughness $W$ (rms deviation of the field fluctuations), which increases with time as $W \sim t^{\beta}$, reaching a saturation value $W_{\text {sat }} \sim L^{\alpha}$ at time $t_{\text {sat }} \sim L^{z}$, where $z=\alpha \beta$ is the dynamic exponent [3] and $\alpha$ is the roughness exponent, related with the fractal dimension of the $u(x)$ profile [8]. The same exponents occur in the two-point statistics, e.g., in the structure factor $S(k, t)=\left\langle|\hat{u}(k, t)|^{2}\right\rangle$, where hat denotes space Fourier transform and $k$ is wave number (two-point correlations in real space are similarly assessed in Ref. [40]). Indeed [8,9], $S(k, t) \sim 1 / k^{2 \alpha+d}$ for $t \gg L^{z}$, while $S(k, t) \sim$ $t^{(2 \alpha+d) / z}$ for $k \ll 1$ (with $d=1$ here). The numerical time evolution of $S(k, t)$ for the various noise conditions is shown in Fig. 1, where the scaling exponents predicted by Yakhot's argument [43] are indeed obtained: Both in the deterministic, and in the conserved-noise cases, Eq. (3) renormalizes into Burgers equation, Eq. (4), with conserved noise $D=$ $0, \tilde{D} \neq 0$, for which $\alpha_{\mathrm{cn}}=-1 / 2$ and $z_{\mathrm{cn}}=3 / 2$ [18]. While $S(k, t)$ approaches the ( $k$-independent) white-noise behavior for increasing $t$ in these two cases, the detailed form of the structure factor curves differs noticeably. In the deterministic case, the scaling exponents are obtained via the integrated $h$ field, trivially expected to scale as $h \sim x^{\alpha+1}[13,18]$. In contrast, for nonconserved noise, Eq. (3) now renormalizes 


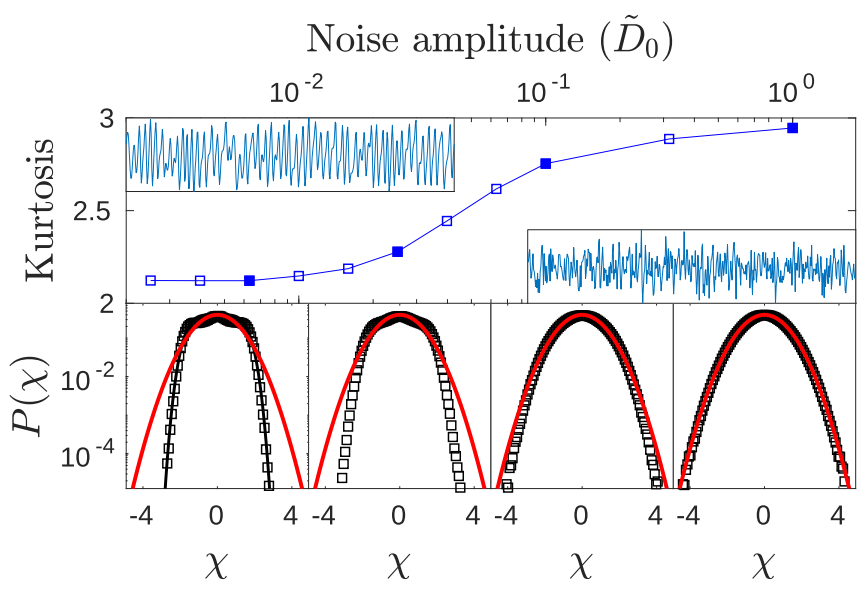

FIG. 2. Top panel: Kurtosis of $u$ fluctuations within the nonlinear regime prior to saturation for Eq. (3) with $D_{0}=0$, and different values of $\tilde{D}_{0}$. The line is a guide to the eye. The bottom panels show the PDF of standardized $u$ fluctuations $(\chi)$ for increasing values of $\tilde{D}_{0}$ left to right, which correspond to the filled squares in the top panel. Red solid lines show an exact Gaussian PDF; the black solid line is for $P[\chi] \sim \exp \left(-\chi^{4.5}\right)$. Insets in top panel show representative $u(x)$ profiles for $\tilde{D}_{0}=0$ (deterministic case; left) and $\tilde{D}_{0}=1$ (conservednoise case; right). Averages are over 10 realizations.

into a noisy Burgers equation, Eq. (4), with nonconserved noise $D \neq 0, \tilde{D}=0$, for which $\alpha_{\mathrm{nc}}=0$ and $z_{\mathrm{nc}}=1$ [46].

The scaling exponent values already imply that the KS equation with nonconserved noise belongs to a different universality class than the deterministic and conserved-noise equations, which share the same values of $\alpha$ and $z$. Hence, for now, we set $D_{0}=0$ and focus on the latter two cases. Still, as noted above, the values of the two independent scaling exponents are currently known $[17,18]$ not to necessarily fix the GSI universality class unambiguously. Moreover, the 1D KPZ universality class also illustrates the fact [10-12] that the field PDF can differ in the nonlinear regime prior to saturation to a steady state, as compared with the PDF of fluctuations around such a steady state after it has been reached. Hence, we next investigate the fluctuation statistics for the $u$ field in Eq. (3) within the nonlinear regime prior to saturation, considering different values of the conserved-noise amplitude $\tilde{D}_{0}$; results are shown in Fig. 2 . In the deterministic $\tilde{D}_{0}=0$ case, the rescaled fluctuations of $u$ around its space average $\bar{u}$, defined as $\chi=(u-\bar{u}) / \operatorname{std}(u)$, exhibit a symmetric probability density function (PDF) whose tails decay much more quickly than those of a Gaussian distribution. Hence, the kurtosis is much smaller than 3; see Fig. 2. This PDF features two symmetric shoulders, implying a relatively high frequency for two characteristic fluctuations in $u$ values, which can be approximately identified by inspection of the $u(x)$ profile shown in the figure. Similar distributions had been earlier reported at steady state [44]. We assess the full time evolution of the skewness and the excess kurtosis of the $u$ fluctuations in Ref. [40], finding them to remain virtually unchanged along the nonlinear time regime. The main qualitative features of the PDF are preserved for increasing values of the conserved-noise amplitude $\tilde{D}_{0}$, up to a certain value. For larger values of $\tilde{D}_{0}$, the fluctuation PDF starts to approach the
Gaussian form, with a kurtosis which approaches the exact Gaussian value; see Fig. 2. Inspection of the representative $u(x)$ profile shown for $\tilde{D}_{0}=1$ indeed suggests the smaller predominance of characteristic fluctuations around the mean than in the deterministic case. Further details on the transition of the PDF with $\tilde{D}_{0}$ are provided in Ref. [40].

While the PDFs of the deterministic and the (large) conserved-noise cases of Eq. (3) are both even function of $\chi$, they are obviously different, especially with respect to the occurrence of "typical" fluctuation values. One could speak of two different subclasses of a single universality class which additionally features $\alpha_{\mathrm{cn}}=-1 / 2$ (white noise) and $z_{\mathrm{cn}}=3 / 2$ (superdiffusive spread of correlations, as in the 1D KPZ equation). However, further dynamical properties suggest that we rather speak of a change in the universality class, with a transition at a well-defined value of $\tilde{D}_{0}$ separating the predominance of chaotic or of stochastic fluctuations. This interpretation is supported by a study of the behavior of the finite-size Lyapunov exponents of the system [56,57] as a function of the conserved-noise amplitude. Specifically, we measure the so-called scale-dependent Lyapunov exponent $\Lambda(\epsilon)$ [57,58], where $\epsilon$ is a distance between trajectories $\left\{T_{i}\left(x_{0}\right)=\left[u\left(x_{0},(i+1) \Delta t\right), u\left(x_{0},(i+\right.\right.\right.$ 2) $\left.\left.\Delta t), \ldots, u\left(x_{0},(i+m) \Delta t\right)\right]\right\}_{i=1}^{I}$ extracted from the time series $u\left(x_{0}, t\right)$, where $x_{0}$ is any fixed value of $x$ and $\Delta t$ is a sampling time. These trajectories are efficient tools to reconstruct the attractor of the dynamics [56]. Once all $(i, j)$ pairs of close-by trajectories are selected for a certain $\epsilon_{0} \ll 1$ such that $0<\left\|T_{i}-T_{j}\right\| / \max _{k, l}\left\|T_{k}-T_{l}\right\|<\epsilon_{0} \ll 1$, we compute $\Lambda\left(\epsilon_{t}\right)=\Delta t^{-1} \ln \epsilon_{t+\Delta t} / \epsilon_{t}$ for each selected $(i, j)$ and for several times $t=N \Delta t$, where $\epsilon_{t}=\left\|T_{i+N}-T_{j+N}\right\|$ and $\epsilon_{t+\Delta t}=$ $\left\|T_{i+N+1}-T_{j+N+1}\right\|$. Results are averaged for different values of $x_{0}$ and, after binning, an $\epsilon$-dependent Lyapunov exponent $\Lambda(\epsilon)$ is obtained. For purely stochastic systems, $\Lambda(\epsilon)$ has been shown to display monotonic power-law decay with $\epsilon$ [58]; in contrast, chaotic systems are characterized by a welldefined plateau where $\Lambda(\epsilon)$ shows $\epsilon$-independent behavior, for not too large $\epsilon$ values [58].

We have studied numerically the behavior of $\Lambda(\epsilon)$ for Eq. (3) and different values of $\tilde{D}_{0}$, setting $D_{0}=0, m=4$, $I=4997$, and $\Delta t=\delta t$; results are shown in Fig. 3. Indeed, in the $\left(\tilde{D}_{0}=0\right)$ deterministic case $\Lambda(\epsilon)$ displays a welldefined plateau for small $\epsilon$, and decays monotonously for $\epsilon \gtrsim 3 \times 10^{-3}$. The plateau width decreases for increasing $\tilde{D}_{0}$. In contrast, for "large" $\tilde{D}_{0}=1, \Lambda(\epsilon)$ decays monotonically with $\epsilon$. We consider that a transition takes place when the plateau first vanishes, for $\tilde{D}_{0} \simeq \tilde{D}_{0, c}=1.6 \times 10^{-3}$. Actually, starting at this value the kurtosis $\mathcal{K}\left(\tilde{D}_{0}\right)$ departs from its deterministic value, approaching Gaussian behavior. Moreover, the $L$ dependence of the threshold value $\tilde{D}_{0, c}$ seems weak; see Fig. 3.

We have additionally assessed this transition between chaotic and stochastic GSI behavior in the KS equation satisfied by the space integral of $u$ defined above, namely,

$$
\partial_{t} h=-v_{0} \partial_{x}^{2} h-\kappa_{0} \partial_{x}^{4} h+\frac{\lambda_{0}}{2}\left(\partial_{x} h\right)^{2}+D_{0} \eta,
$$

where we now only consider the nonconserved noise case, relevant within Yakhot's argument [16,43,47]. In Fig. 4, we confirm that, for an increasing noise amplitude $D_{0}, \Lambda(\epsilon)$ be- 


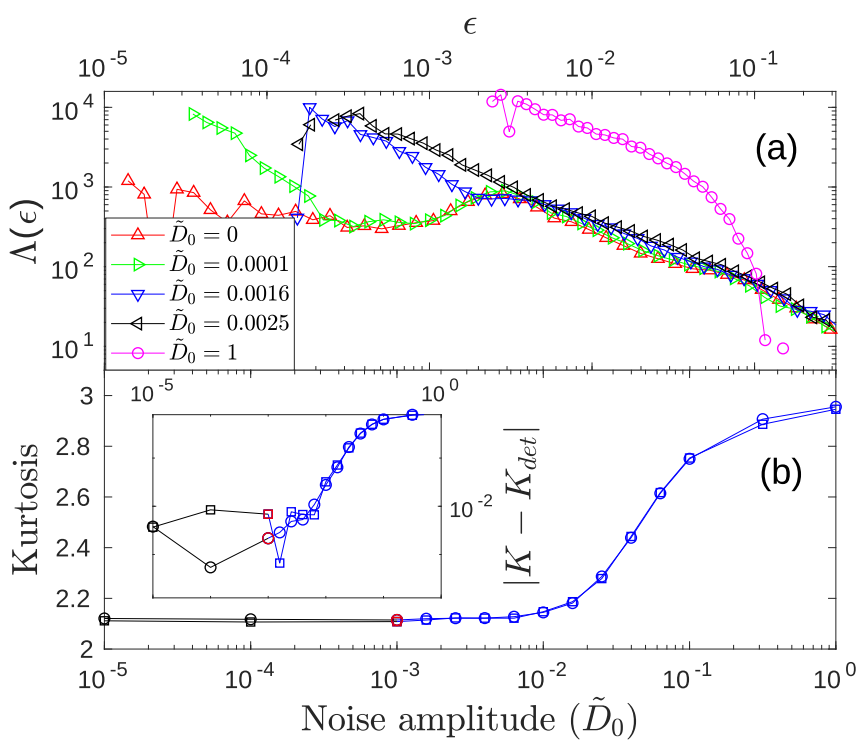

FIG. 3. (a) Scale-dependent Lyapunov exponent vs $\epsilon$ from numerical solutions of Eq. (3) within the nonlinear regime prior to saturation, for $D_{0}=0$ and conserved-noise amplitudes $\tilde{D}_{0}$ as in the legend. (b) Kurtosis of $u$ fluctuations vs $\tilde{D}_{0}$ for $L=1024$ (squares) and $L=4096$ (circles). Inset: Distance between $\mathcal{K}\left(\tilde{D}_{0}\right)$ and the kurtosis of the deterministic system, $\mathcal{K}_{\text {det }}$, vs $\tilde{D}_{0}$. For each $L$, the difference grows (blue data) to the right of the corresponding red point. All lines are guides to the eye. Averages are over 10 realizations.

haves similarly to Eq. (3): The $\left(D_{0}=0\right)$ deterministic system shows a plateau of $\epsilon$-independent behavior which disappears for $D_{0} \gtrsim 0.01$, beyond which stochastic behavior ensues. However, now the transition does not reflect into a change between two different fluctuation PDFs. Indeed, in the chaotic case $\left(D_{0}=0\right)$ fluctuations of Eq. (5) are known to be TracyWidom distributed in the nonlinear regime prior to saturation [16], while Fig. 4 indicates that so do fluctuations for a "large" value of stochastic noise. Scaling exponent values are known

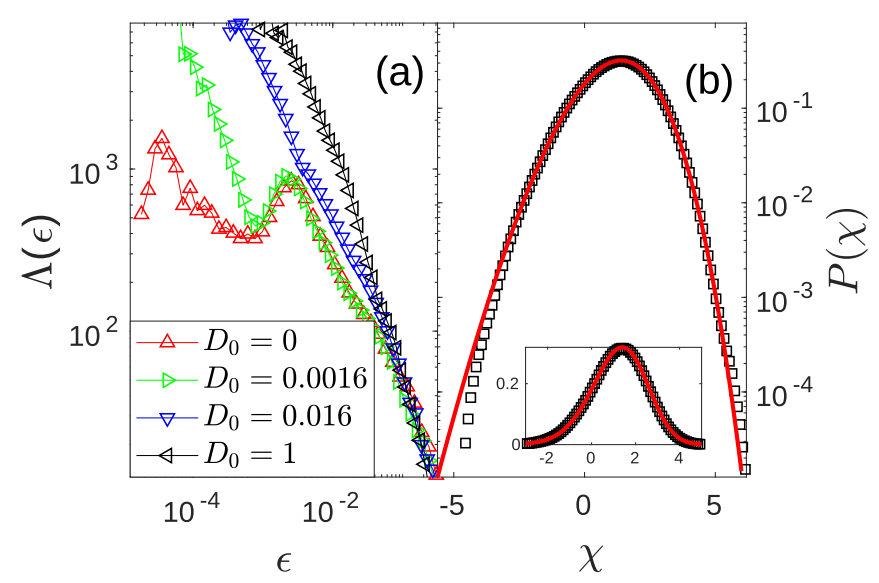

FIG. 4. (a) Scale-dependent Lyapunov exponent for solutions of the KS Eq. (5) within nonlinear regime prior to saturation, for noise amplitudes $D_{0}$ as in the legend. Remaining parameters as in previous figures. (b) PDF of $h$ fluctuations within nonlinear regime for $D_{0}=$ 1. Red solid line shows the exact GOE-TW PDF [10-12]. Inset: same data in a linear plot. Averages are over 10 realizations. to be $1 \mathrm{D} \mathrm{KPZ}$, independently of the value of $D_{0}[16,48]$. For a graphical summary of these results and those obtained for Eq. (3), see Fig. S6 of Ref. [40].

In summary, we have seen that Yakhot's classic argument on the asymptotic equivalence between SC and GSI holds only partly for Eqs. (3) and (5); specifically, for the conserved KS equation, Eq. (3), different universality classes occur with different field PDF, albeit with the same scaling exponent values. This fact is not captured by Yakhot's argument which, while incorporating the basic system symmetries (conserved vs nonconserved dynamics, etc.), misses key differences between PDFs which are otherwise consistent with the former. The dynamical role of symmetries can be subtle indeed in the present class of nonequilibrium critical systems [4,46,59].

Crucially, each one of the GSI universality classes occurring in Eq. (3) correlates with the nature (chaotic or stochastic) of the mechanism controlling fluctuations in the system, with the transition between them nontrivially occurring at a nonzero stochastic noise amplitude. This transition could be experimentally verified, for instance, in epitaxial growth of vicinal surfaces [36], where the KS equation describes the dynamics of atomic steps separating terraces under nonnegligible adatom desorption [35]. In such a case, the equation for the step slope is Eq. (3), where $\tilde{D}_{0}$ scales as an inverse power of the characteristic desorption time.

For the nonconserved KS equation, Eq. (5), although an analogous transition takes place in the dominance of chaotic or stochastic fluctuations, on both sides of the transition the field PDF and the scaling exponent are those of the 1D KPZ universality class. Indeed, the TW distribution is not only relevant to the stochastic $1 \mathrm{D} \mathrm{KPZ}$ class but also describes the fluctuations of deterministic chaotic systems [60]. This coincidence might well be accidental and limited to 1D systems. Its exploration in 2D might provide some clue on the relation between the deterministic KS and the stochastic KPZ equations in higher dimensions [61], an open challenge in the fundamental understanding of spatiotemporal chaos [62].

With respect to the specifics of the present transition, it would be interesting to obtain analytical estimates on the threshold noise amplitude and to assess nontrivial consequences on physical quantities beyond the field PDF. The behavior discussed above for $S(k, t)$ already indicates differences in the equal-time two-point statistics, but two-time statistics may introduce additional novelties. In this process, it would be interesting to find analogous transitions, but in which the chaotic and stochastic "phases" might also differ by the values of the scaling exponents.

Finally, the correlation between the field PDF and the nature of the fluctuations underscores the importance of assessing the PDF explicitly, to correctly identify the GSI universality class. This is particularly critical in view of the plethora of experimental complex systems that can be described by a paradigmatic model like the $\mathrm{KS}$ equation, in its different (conserved or nonconserved; deterministic or stochastic) forms, especially when both chaotic and stochastic fluctuations may be operative at comparable space-time scales.

This work has been supported by Ministerio de Economía y Competitividad, Agencia Estatal de 
Investigación, and Fondo Europeo de Desarrollo Regional (Spain and European Union) through Grant No. PGC2018094763-B-I00. E.R.-F. also acknowledges financial support by Ministerio de Educación, Cultura y Deporte (Spain) through Formación del Profesorado Universitario Scholarship No. FPU16/06304.
[1] G. Grinstein, Generic scale invariance and self-organized criticality, in Scale Invariance, Interfaces, and Non-equilibrium Dynamics, edited by A. McKane, M. Droz, J. Vannimenus, and D. Wolf (Springer, Cambridge, UK, 1995).

[2] J. P. Sethna, Statistical Mechanics: Entropy, Order Parameters, and Complexity (Oxford University Press, New York, 2006).

[3] U. C. Täuber, Critical Dynamics (Cambridge University Press, Cambridge, UK, 2014).

[4] L. M. Sieberer, S. D. Huber, E. Altman, and S. Diehl, Dynamical Critical Phenomena in Driven-Dissipative Systems, Phys. Rev. Lett. 110, 195301 (2013).

[5] L. M. Sieberer, and E. Altman, Topological Defects in Anisotropic Driven Open Systems, Phys. Rev. Lett. 121, 085704 (2018).

[6] M. A. Munoz, Colloquium: Criticality and dynamical scaling in living systems, Rev. Mod. Phys. 90, 031001 (2018).

[7] M. Perc, J. J. Jordan, D. G. Rand, Z. Wang, S. Boccaletti, and A. Szolnoki, Statistical physics of human cooperation, Phys. Rep. 687, 1 (2017).

[8] A.-L. Barabási and H. E. Stanley, Fractal Concepts in Surface Growth (Cambridge University Press, Cambridge, UK, 1995).

[9] J. Krug, Origins of scale invariance in growth processes, Adv. Phys. 46, 139 (1997).

[10] T. Kriecherbauer and J. Krug, A pedestrian's view on interacting particle systems, KPZ universality, and random matrices, J. Phys. A: Math. Theor. 43, 403001 (2010).

[11] T. Halpin-Healy and K. A. Takeuchi, A KPZ cocktail—shaken, not stirred..., J. Stat. Phys. 160, 794 (2015).

[12] K. A. Takeuchi, An appetizer to modern developments on the Kardar-Parisi-Zhang universality class, Physica A (Amsterdam, Neth.) 504, 77 (2018).

[13] M. Kardar, G. Parisi, and Y. C. Zhang, Dynamic Scaling of Growing Interfaces, Phys. Rev. Lett. 56, 889 (1986).

[14] L. Chen, C. F. Lee, and J. Toner, Mapping two-dimensional polar active fluids to two-dimensional soap and one-dimensional sandblasting, Nat. Commun. 7, 12215 (2016).

[15] A. Nahum, J. Ruhman, S. Vijay, and J. Haah, Quantum Entanglement Growth Under Random Unitary Dynamics, Phys. Rev. X 7, 031016 (2017).

[16] D. Roy and R. Pandit, One-dimensional Kardar-Parisi-Zhang and Kuramoto-Sivashinsky universality class: Limit distributions, Phys. Rev. E 101, 030103(R) (2020).

[17] Y. Saito, M. Dufay, and O. Pierre-Louis, Nonequilibrium Cluster Diffusion During Growth and Evaporation in Two Dimensions, Phys. Rev. Lett. 108, 245504 (2012).

[18] E. Rodriguez-Fernandez and R. Cuerno, Non-KPZ fluctuations in the derivative of the Kardar-Parisi-Zhang equation or noisy Burgers equation, Phys. Rev. E 101, 052126 (2020).

[19] I. S. S. Carrasco, K. A. Takeuchi, S. C. Ferreira, and T. J. Oliveira, Interface fluctuations for deposition on enlarging flat substrates, New J. Phys. 16, 123057 (2014).

[20] Y. T. Fukai and K. A. Takeuchi, Kardar-Parisi-Zhang Interfaces With Inward Growth, Phys. Rev. Lett. 119, 030602 (2017).
[21] I. S. S. Carrasco and T. J. Oliveira, Kardar-Parisi-Zhang growth on one-dimensional decreasing substrates, Phys. Rev. E 98, 010102(R) (2018).

[22] S. N. Santalla, J. Rodríguez-Laguna, A. Celi, and R. Cuerno, Topology and the Kardar-Parisi-Zhang universality class, J. Stat. Mech.: Theory Exp. (2017) 023201.

[23] I. S. S. Carrasco and T. J. Oliveira, Circular Kardar-ParisiZhang interfaces evolving out of the plane, Phys. Rev. E 99 , 032140 (2019).

[24] I. S. S. Carrasco and T. J. Oliveira, Geometry dependence in linear interface growth, Phys. Rev. E 100, 042107 (2019).

[25] I. S. S. Carrasco and T. J. Oliveira, Universality and geometry dependence in the class of the nonlinear molecular beam epitaxy equation, Phys. Rev. E 94, 050801(R) (2016).

[26] Y. Kuramoto and T. Tsuzuki, Persistent propagation of concentration waves in dissipative media far from thermal equilibrium, Prog. Theor. Phys. 55, 356 (1976).

[27] D. M. Michelson and G. I. Sivashinsky, Nonlinear analysis of hydrodynamic instability in laminar flames, II. Numerical experiments, Acta Astron. 4, 1207 (1977).

[28] M. C. Cross and P. C. Hohenberg, Pattern formation outside of equilibrium, Rev. Mod. Phys. 65, 851 (1993).

[29] M. C. Cross and P. C. Hohenberg, Spatiotemporal chaos, Science 263, 1570 (1994).

[30] J. Pathak, Z. Lu, B. Hunt, M. Girvan, and E. Ott, Using machine learning to replicate chaotic attractors and calculate Lyapunov exponents from data, Chaos 27, 121102 (2017).

[31] J. Pathak, B. Hunt, M. Girvan, Z. Lu, and E. Ott, Model-Free Prediction of Large Spatiotemporally Chaotic Systems from Data: A Reservoir Computing Approach, Phys. Rev. Lett. 120, 024102 (2018).

[32] A. A. Nepomnyashchii, Stability of wavy conditions in a film flowing down an inclined plane, Fluid Dyn. 9, 354 (1974).

[33] For a stochastic generalization with $D_{0} \neq 0, \tilde{D}_{0}=0$, see, e.g., M. Pradas, G. A. A. Pavliotis, S. Kalliadasis, D. T. T. Papageorgiou, and D. Tseluiko, Additive noise effects in active nonlinear spatially extended systems, Eur. J. Appl. Math. 23, 563 (2012).

[34] C. Misbah, H. Müller-Krumbhaar, and D. E. Temkin, Interface structure at large supercooling, J. Phys. I 1, 585 (1991).

[35] A. Karma and C. Misbah, Competition between Noise and Determinism in Step Flow Growth, Phys. Rev. Lett. 71, 3810 (1993).

[36] C. Misbah, O. Pierre-Louis, and Y. Saito, Crystal surfaces in and out of equilibrium: A modern view, Rev. Mod. Phys. 82, 981 (2010).

[37] R. Cuerno and A.-L. Barabási, Dynamic Scaling of IonSputtered Surfaces, Phys. Rev. Lett. 74, 4746 (1995).

[38] K. B. Lauritsen, R. Cuerno, and H. A. Makse, Noisy KuramotoSivashinsky equation for an erosion model, Phys. Rev. E 54, 3577 (1996). 
[39] R. Cuerno and M. Castro, Transients Due to Instabilities Hinder Kardar-Parisi-Zhang Scaling: A Unified Derivation for Surface Growth by Electrochemical and Chemical Vapor Deposition, Phys. Rev. Lett. 87, 236103 (2001).

[40] See Supplemental Material at http://link.aps.org/supplemental/ 10.1103/PhysRevResearch.3.L012020 for a derivation of the stochastic KS equation with conserved noise for a falling liquid film and for additional numerical results on Eqs. (3) and (5).

[41] R. Seemann, S. Herminghaus, and K. Jacobs, Dewetting Patterns and Molecular forces: A Reconciliation, Phys Rev. Lett. 86, 5534 (2001).

[42] K. Mecke and M. Rauscher, On thermal fluctuations in thin film flow, J. Phys. Condens. Matter 17, S3515 (2005).

[43] V. Yakhot, Large-scale properties of unstable systems governed by the Kuramoto-Sivashinksi equation, Phys. Rev. A 24, 642 (1981).

[44] F. Hayot, C. Jayaprakash, and C. Josserand, Long-wavelength properties of the Kuramoto-Sivashinsky equation, Phys. Rev. E 47, 911 (1993).

[45] D. Forster, D. R. Nelson, and M. J. Stephen, Large-distance and long-time properties of a randomly stirred fluid, Phys. Rev. A 16, 732 (1977).

[46] E. Rodriguez-Fernandez and R. Cuerno, Gaussian statistics as an emergent symmetry of the stochastic scalar Burgers equation, Phys. Rev. E 99, 042108 (2019).

[47] K. Sneppen, J. Krug, M. H. Jensen, C. Jayaprakash, and T. Bohr, Dynamic scaling and crossover analysis for the KuramotoSivashinsky equation, Phys. Rev. A 46, R7351(R) (1992).

[48] R. Cuerno, H. A. Makse, S. Tomassone, S. T. Harrington, and H. E. Stanley, Stochastic Model for Surface Erosion Via Ion Sputtering: Dynamical Evolution from Ripple Morphology to Rough Morphology, Phys. Rev. Lett. 75, 4464 (1995).

[49] K. Ueno, H. Sakaguchi, and M. Okamura, Renormalizationgroup and numerical analysis of a noisy Kuramoto-Sivashinsky equation in $1+1$ dimensions, Phys. Rev. E 71, 046138 (2005).

[50] H. W. Xi, R. Toral, J. D. Gunton, and M. I. Tribelsky, Extensive chaos in the Nikolaevskii model, Phys. Rev. E 62, R17(R) (2000).
[51] L. Giada, A. Giacometti, and M. Rossi, Pseudospectral method for the Kardar-Parisi-Zhang equation, Phys. Rev. E 65, 036134 (2002).

[52] R. Gallego, M. Castro, and J. M. López, Pseudospectral versus finite-difference schemes in the numerical integration of stochastic models of surface growth, Phys. Rev. E 76, 051121 (2007).

[53] M. Nicoli, M. Castro, and R. Cuerno, Unified moving-boundary model with fluctuations for unstable diffusive growth, Phys. Rev. E 78, 021601 (2008).

[54] R. Toral and P. Colet, Stochastic Numerical Methods: An Introduction for Students and Scientists (Wiley-VCH, Weinheim, 2014).

[55] R. Gallego, Predictor-corrector pseudospectral methods for stochastic partial differential equations with additive white noise, Appl. Math. Comput. 218, 3905 (2011).

[56] A. Pikovsky and A. Politi, Lyapunov Exponents: A Tool to Explore Complex Dynamics (Cambridge University Press, Cambridge, UK, 2016).

[57] M. Cencini and A. Vulpiani, Finite size Lyapunov exponent: Review on applications, J. Phys. A: Math. Theor. 46, 254019 (2013).

[58] J. B. Gao, J. Hu, W. W. Tung, and Y. H. Cao, Distinguishing chaos from noise by scale-dependent Lyapunov exponent, Phys. Rev. E 74, 066204 (2006).

[59] S. Mathey, E. Agoritsas, T. Kloss, V. Lecomte, and L. Canet, Kardar-Parisi-Zhang equation with short-range correlated noise: Emergent symmetries and nonuniversal observables, Phys. Rev. E 95, 032117 (2017).

[60] H. Spohn, in Thermal Transport in Low Dimensions, Lecture Notes in Physics Vol. 921, edited by S. Lepri (Springer International, Cham, 2016), Chap. 3, pp. 107-158.

[61] P. Manneville and H. Chaté, Phase turbulence in the twodimensional complex Ginzburg-Landau equation, Physica D (Amsterdam, Neth.) 96, 30 (1996).

[62] B. M. Boghosian, C. C. Chow, and T. Hwa, Hydrodynamics of the Kuramoto-Sivashinsky Equation in two Dimensions, Phys. Rev. Lett. 83, 5262 (1999). 